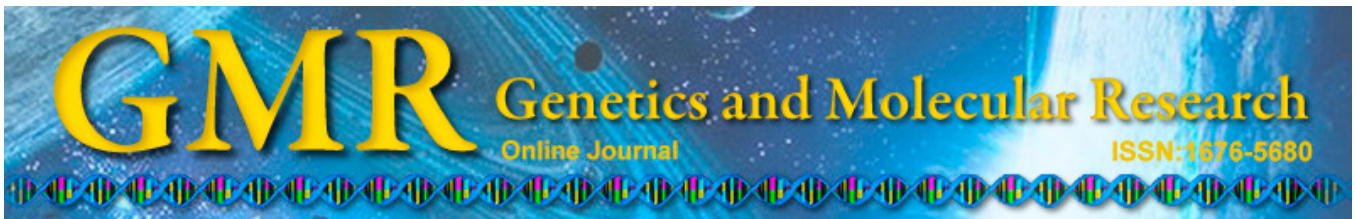

\title{
Treatment of moyamoya disease by multipoint skull drilling for indirect revascularization combined with mobilization of autologous bone marrow stem cells
}

\author{
R. Wu ${ }^{1}$, N. Su ${ }^{1}$, Z. Zhang ${ }^{1}$ and F. Jia ${ }^{2}$ \\ ${ }^{1}$ Department of Neurosurgery, \\ Inner Mongolia Autonomous Region People's Hospital, Hohhot, China \\ ${ }^{2}$ Department of Neurosurgery, \\ Shanghai Renji Hospital, School of Medicine, Shanghai JiaoTong University, \\ Shanghai, China \\ Corresponding author: F. Jia \\ E-mail: jiafengdn@126.com \\ Genet. Mol. Res. 14 (3): 7519-7528 (2015) \\ Received November 3, 2014 \\ Accepted January 26, 2015 \\ Published July 3, 2015 \\ DOI http://dx.doi.org/10.4238/2015.July.3.27
}

\begin{abstract}
This study discusses the clinical efficacy of multipoint skull drilling for indirect revascularization combined with mobilization of autologous bone marrow stem cells and use of simvastatin in the treatment of moyamoya disease. Seventy-eight patients [control group (group A), 39 patients; experimental group (group B), 39 patients] with moyamoya disease were selected. Group A underwent indirect revascularization, and group B, in addition to indirect revascularization, received alternate subcutaneous injections from day 7 post-surgery. The number and differentiation of the mobilized bone marrow stem cells were detected by the proportion of hematopoietic progenitor cell (HPCs) in mononuclear cells (MNCs) in the peripheral blood. There was no statistical difference in the BI $(80.2 \pm 13.7)$ and NIHSS $(6.7 \pm$ $2.3)$ scores between the groups before treatment $(\mathrm{P}>0.05)$. The CSS
\end{abstract}


score of group B was $13.5 \pm 0.6$ and there was a statistical significance compared to group A $(18.2 \pm 0.8)(\mathrm{P}<0.05)$. There was no statistical difference in the proportion of CD34+ CD133+ cells in MNCs in peripheral blood before surgery between the two groups $(\mathrm{P}>0.05)$ and the proportions of $\mathrm{CD} 34+\mathrm{CD} 133+$ cells in MNCs in peripheral blood in groups $\mathrm{A}$ and $\mathrm{B}$ at 30 days after surgery were significantly higher than those before surgery $(\mathrm{P}<0.05)$. Treating moyamoya disease by multipoint skull drilling for indirect revascularization combined with mobilization of autologous bone marrow stem cells and simvastatin is a safe and effective method as it can promote recovery of neurological functions and improve patients' daily living abilities and quality of life.

Key words: Bone marrow stem cell; Simvastatin; Moyamoya disease; Multipoint skull drilling; Indirect revascularization

\section{INTRODUCTION}

Moyamoya disease (MMD) is a rare, progressive, occlusive cerebrovascular disease caused by an unexplained abnormal vascular network at the base of the brain. It is associated with high fatality and disability rates and lacks specific medical intervention. In recent years, surgical revascularization has achieved substantial progress in MMD treatment (Kim et al., 2013; Skeik et al., 2013). Previous research has found that bone marrow stem cells (BMSCs) have the potential to differentiate into multiple cells. They can differentiate into neural, neuroglial, and vascular endothelial cells, among others. They can help protect injured nerves and accelerate recovery of impaired functions (Lee et al., 2012; Takemoto et al., 2012). Statins can mobilize bone marrow endothelial stem cells and promote recovery from nerve injury. Mobilization of autologous BMSCs has improved treatment of ischemic cerebrovascular diseases to a certain extent (Almansob et al., 2012; Uhiara et al., 2012). Vasculogenesis and neurogenesis are two major pathophysiologic processes that occur in the brain after cerebral ischemia. Both play an important role in the recovery of function after cerebral ischemic injury (Wang et al., 2012; Fujimoto et al., 2013). Patients with MMD and cerebral ischemic injury can be treated by surgical intervention combined with stem cell mobilization. This therapy can promote vasculogenesis and nerve regeneration, reduce apoptosis of cells in the cerebral ischemic lesions, and improve brain function (Xu et al., 2012; Cho et al., 2013).

This study aimed to evaluate the safety and efficacy of indirect revascularization combined with mobilization of autologous stem cells and simvastatin administration in the treatment of MMD.

\section{MATERIAL AND METHODS}

\section{Clinical data}

We selected 78 patients [37 men, 41 women; average age, 42.8 (range, 20-55) years] with MMD and complete data available, who underwent skull drilling for indirect revascularization in our hospital from October 2006 to February 2013.

The inclusion criteria were as follows: 1) symptoms of regional or diffuse neuro- 
logical injury, evidentiary imaging material, and a definite clinical diagnosis of MMD; 2) no severe complications such as malignant tumors and myocardial infarction; and 3) signed informed consent obtained from patient and family members for the therapeutic regimen.

\section{Therapeutic intervention}

The patients were divided into two groups according to the therapeutic regimen. In the control group (group A), the 39 patients were treated with indirect revascularization. Multipoint skull drilling was performed without damaging the periosteum at sites corresponding to the blood supply region of the diseased blood vessels as indicated by duro-arteriosynangiosis (DAS) after routine drug therapy. The endocranium at the site was excised. The bone holes were filled with gelatin sponge after opening the arachnoid. The drilling sites were in the frontal portion corresponding to the anterior cerebral artery and the top portion corresponding to the middle cerebral artery. The posterior cerebral artery corresponded with the occiput.

Those in the experimental group were subjected to alternate subcutaneous injection of $2.5 \mu \mathrm{g} / \mathrm{kg}$ recombinant human granulocyte-colony stimulating factor (rhG-CSF) and recombinant human granulocyte macrophage-colony stimulating factor (rhGM-CSF) (GeneTech Biotechnology Development Company Limited; China), once every 3 days, for 30 consecutive days. Routine blood and coagulation function reexaminations were performed before each use of the mobilization agent. If the white blood cell count rose to $<20 \times 10^{9} / \mathrm{L}$, the use of mobilization agent was continued; if the white blood cell count was $\geq 20 \times 10^{9} / \mathrm{L}$, the injection was suspended. The injection was administered in accordance with the reexamination result on the following day. The hepatic and renal functions were re-examined after one course of treatment. Meanwhile, $10 \mathrm{mg} / \mathrm{d}$ simvastatin was administered orally from the 3 rd day after the surgery, for 30 consecutive days. The liver functions were examined each week during treatment.

\section{Evaluation indicators for therapeutic effects}

Recovery of basic activities of daily living was evaluated based on the Barthel Index (BI). The Chinese Stroke Scale (CSS) and National Institutes of Health Stroke Scale (NIHSS) were used for evaluation of neurologic impairment (Fujimura et al., 2012; Gu et al., 2012).

The NIHSS components included level of consciousness, gaze, visual field, facial paralysis, upper limb movement, lower limb movement, limb ataxia, sensation, best language, dysarthria, regression, and inattention.

The number of CD-, were determined with the flow cytometer. The number and differentiation of the mobilized BMSCs were evaluated by detecting the proportion of HPCs in Mononuclear Cells(MNCs) in the peripheral blood. Occurrence of any toxic effects and side effects was closely monitored during treatment.

\section{Statistical methods}

SPSS 15.0 (IBM,US.) was used for statistical analysis. The data are reported as means \pm standard deviation (means \pm SD). Pairwise comparisons were made within the group. A paired data F-test was conducted for statistical analysis between the two groups. $\mathrm{P}<0.05$ indicated a statistically significant difference. 


\section{RESULTS}

\section{BI}

There was no statistical difference in the BI between the two groups before treatment $(\mathrm{P}>0.05)$. The $\mathrm{BI}$ of groups $\mathrm{A}$ and $\mathrm{B}$ at 1,3 , and 6 months after treatment exhibited an increasing trend; the $\mathrm{BI}$ of groups $\mathrm{A}$ and $\mathrm{B}$ increased in sequence and there was a significant difference at the same point in time $(\mathrm{P}<0.05)$ (Figure 1 and Table 1).

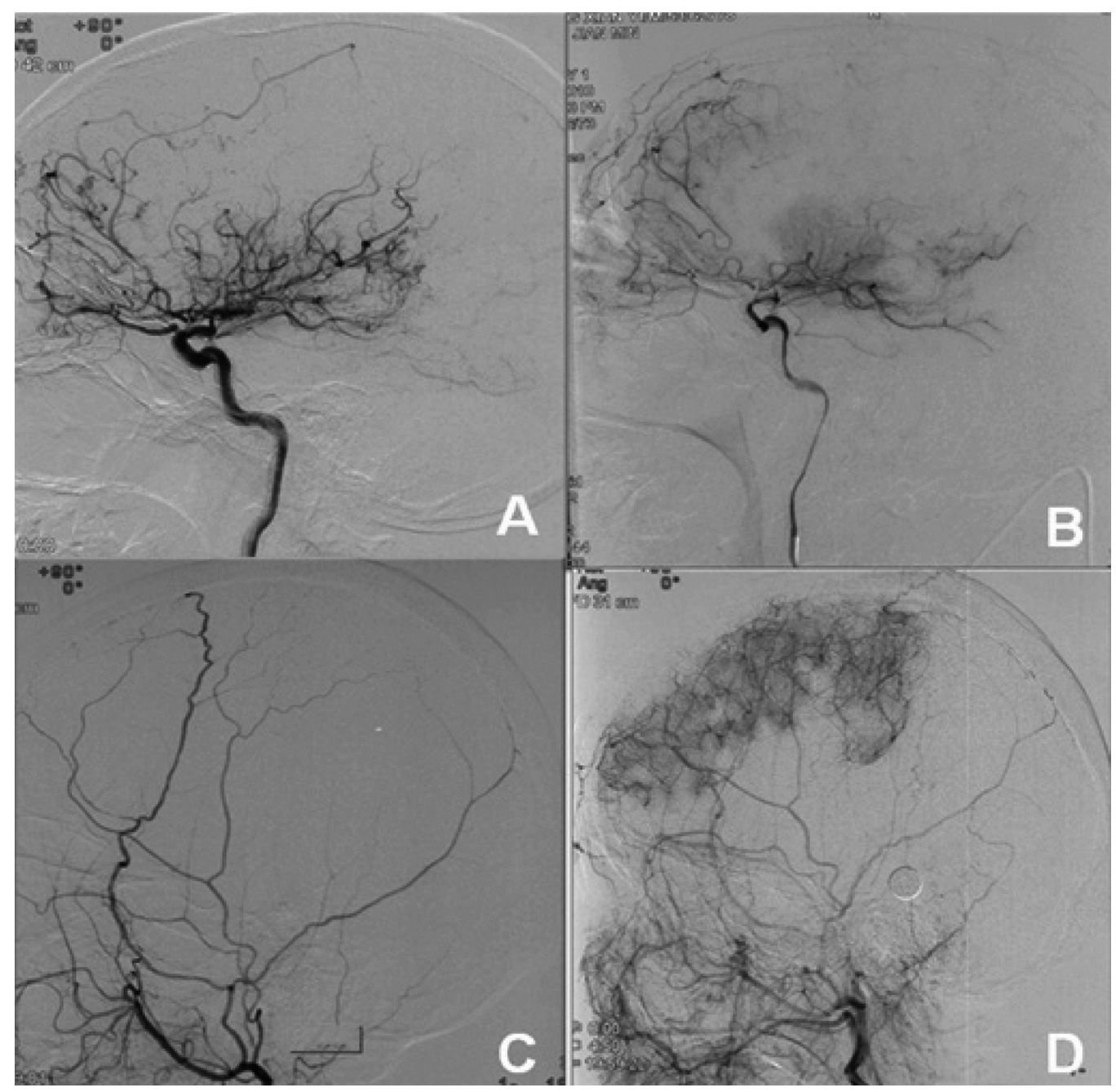

Figure 1. Duro-arteriosynangiosis (DSA) before and after indirect revascularization (multipoint skull drilling) for moyamoya disease; A: Typical moyamoya-like blood vessels on a lateral radiograph of the internal carotid artery before the surgery; B: Moyamoya-like blood vessels were significantly decreased during re-examination at 12 months post-surgery; C: Lateral radiograph of ipsilateral external carotid artery before the surgery; D: Extensive new compensatory blood vessels in the skull drilling regions at 12 months after the surgery. 
Table 1. Barthel indexes at different points in time in both groups.

\begin{tabular}{lcccc}
\hline Group & Before treatment & 1 month after treatment & 3 months after treatment & 6 months after treatment \\
\hline Control group (group A) & $26.7 \pm 8.4$ & $38.2 \pm 11.6$ & $57.3 \pm 11.8$ & $71.6 \pm 12.9$ \\
Experimental group (group B) & $25.6 \pm 9.1$ & $44.1 \pm 11.8^{*}$ & $69.1 \pm 12.7^{*}$ & $80.2 \pm 13.7^{*}$ \\
\hline
\end{tabular}

Compared with the control group, $* \mathrm{P}<0.05$.

\section{NIHSS score}

There was no statistically significant difference in the NIHSS scores between the two groups before treatment $(\mathrm{P}>0.05)$. The NIHSS scores of groups A and B exhibited a decreasing trend at 6 and 12 months after treatment $(\mathrm{P}<0.05)$; there were significant statistical differences between groups A and B at 6 and 12 months after the surgery $(\mathrm{P}<0.05)$ (Table 2).

Table 2. NIHSS scores at different points in time in both groups.

\begin{tabular}{lccr}
\hline Group & Before treatment & 6 months after treatment & 12 months after treatment \\
\hline Control group (group A) & $24.7 \pm 3.8$ & $15.6 \pm 3.1^{*}$ & $10.6 \pm 2.7^{*}$ \\
Experimental group (group B) & $24.2 \pm 3.6$ & $12.3 \pm 2.8^{* \#}$ & $6.7 \pm 2.3^{* \#}$ \\
\hline
\end{tabular}

Compared to the treatment in the same group, ${ }^{*} \mathrm{P}<0.05$; compared to the control group in the same period, ${ }^{\#} \mathrm{P}<0.05$.

\section{CSS score}

The CSS score decreased slightly 1 month after treatment in both groups, and the CSS score decreased significantly in group B compared with group A $(\mathrm{P}<0.05)$. Three months after treatment, there was a statistical significance compared with group $\mathrm{A}(\mathrm{P}<0.05)($ Table 3$)$.

Table 3. CSS scores before and after treatment in patients with ischemic cerebrovascular diseases.

\begin{tabular}{lccr}
\hline Group & Before treatment & 1 month after treatment & 3 months after treatment \\
\hline Control group (group A) & $31.5 \pm 1.8$ & $24.7 \pm 1.1^{*}$ & $18.2 \pm 0.8^{*}$ \\
Experimental group (group B) & $30.7 \pm 1.8$ & $20.6 \pm 1.0^{* \#}$ & $13.5 \pm 0.6^{* \#}$ \\
\hline
\end{tabular}

Compared to the treatment in the same group, ${ }^{*} \mathrm{P}<0.05$; compared to the control group in the same period, ${ }^{*} \mathrm{P}<0.05$.

\section{CD34+ CDI33+ cell counting}

There was no statistical difference $(\mathrm{P}>0.05)$ in the proportion of $\mathrm{CD} 34+\mathrm{CD} 133+$ cells in MNCs in peripheral blood before surgery between the two groups $(\mathrm{P}>0.05)$. The proportions in groups $\mathrm{A}$ and $\mathrm{B}$ at 30 days after the surgery were significantly higher than those before surgery $(\mathrm{P}<0.05)$. The proportion in group $\mathrm{A}$ was significantly higher than that in group B $(\mathrm{P}<0.05)$ (Table 4).

Table 4. CD34+ CD133+ cell count in both groups before and after treatment (\% MNC).

\begin{tabular}{lcc}
\hline Group & Before treatment & 30 days after treatment \\
\hline Control group (group A) & $0.34 \pm 0.07$ & $2.06 \pm 0.11^{*}$ \\
Experimental group (group B) & $0.32 \pm 0.08$ & $3.67 \pm 0.19^{* \#}$ \\
\hline
\end{tabular}

Compared to the treatment in the same group, ${ }^{*} \mathrm{P}<0.05$; compared to the control group in the same period ${ }^{\#} \mathrm{P}<0.05$. 


\section{Clinical toxic and side effects}

No significant adverse effects were experienced by the patients during the entire study; 4 patients in group B suffered from slightly abnormal coagulation function, 1 patient suffered from fever (temperature $<39^{\circ} \mathrm{C}$ ), and 2 patients suffered from muscle and joint pain in all four limbs. All patients recovered within 2 days after rhG-CSF/rhGM-CSF was discontinued. No specific abnormal conditions, deteriorated limb sensation and motor functions, respiratory dysfunction, cerebral hemorrhage, cerebral infarction, or abnormal mental state were present during the follow-up visits in any of the patients.

\section{DISCUSSION}

MMD primarily manifests as severe stenosis or occlusion of the terminal internal carotid artery, middle cerebral artery, and proximal end of the anterior cerebral artery, and significant endangium incrassation (Motoshima et al., 2012; Tseng and Luo, 2013). The clinical manifestations include insufficient cerebral perfusion, transient cerebral ischemia, cerebral apoplexy, and epilepsy (Choi et al., 2012; Southerland et al., 2013). Focal neurofunctional deficit within the dominated region of the diseased blood vessels after cerebral ischemia severely affects the patients' quality of life. Vasculogenesis and neurogenesis are two major pathophysiologic processes that occur in the brain after cerebral ischemia. Both play an important role in recovery of function after cerebral ischemic injury (Heijmen et al., 2012; Sasaki et al., 2012). Drug therapy is not very effective in the treatment of this disease. Therefore, surgical revascularization is the primary treatment intervention for MMD. MMD largely manifests as cerebral hemorrhage in adults and cerebral ischemia in children. The cerebral hemorrhage rate in adult patients with MMD is over $50 \%$ and in children is only $5 \%$ (Kainth et al., 2013). However, clinically, the therapeutic strategies for the two different pathophysiologic processes are usually the same. The main approach is to build a circulation path from the extracranial arteries to the brain surface. The common surgical methods include direct revascularization and indirect revascularization. Some clinicians have effectively combined in the treatment of MMD and emphasized the roles of temporalis, dura mater, and superficial temporal artery (STA) in blood supply. The symptoms of cerebral ischemia and epilepsy are alleviated significantly in most patients after successful implementation of revascularization (Bao et al., 2012; Dorfman et al., 2012).

The therapeutic effect of indirect revascularization in MMD has been widely accepted. Separate skull drilling can promote formation of collateral vessels at the ischemic site. Based on the blood supply site of the diseased blood vessels indicated by DSA, multiple holes are drilled at corresponding sites to slightly expand the bone window and remove the endocranium. The drilling sites were in the frontal portion corresponding to the anterior cerebral artery and the top portion corresponding to the middle cerebral artery. The posterior cerebral artery corresponded with the occiput. The multi-point skull drilling technique is characterized by ease of performance and few intraoperative and postoperative complications. The drilling positions and number of holes depend on the region of cerebral ischemia (Miyawaki et al., 2012; Volkan-Salanci et al., 2012; Liu et al., 2013). Thus, it is not restricted to the blood supply regions for the middle cerebral artery. Collateral circulation can also be established for the ischemic regions of the anterior and posterior cerebral arteries. The results of the surgery are reliable and the patients' symptoms can be significantly improved after the surgery. Thus, it can serve as a separate surgical method or as an adjuvant therapy for anastomoses of other 
blood vessels (Mishra et al., 2012). Our study demonstrated improvement in the BI, NIHS, and CSS scores 1 month after treatment in 39 group A patients; the BI, NIHSS, and CSS scores at 3 months after treatment were better than those before treatment and the activities of daily living were significantly improved. Our results have shown that indirect revascularization can improve blood supply in the ischemic cerebral region, reduce cerebral injury in the ischemic lesion, and promote recovery of brain functions (Yonekawa, 2012).

Previous research has indicated that the brain has a self-repair ability. There are neural stem cells with self-renewal and differentiative potential within the subventricular zone and hippocampus dentate gyrus subgranular zone of the adult brain. BMSCs constitute a complex cell population that includes hematopoietic stem cells, mesenchymal stem cells, vascular endothelial progenitor cells, macrophages, and lymphocytes, among others. Previous studies have found that BMSCs play a role in protecting nerves and promoting nerve regeneration. rhGM-CSF and rhG-CSF are powerful mobilization agents for BMSCs. They can substantially raise the number of stem cells in peripheral blood, which migrate towards the cerebral ischemic site. They facilitate nerve cell differentiation in a specific pathological environment of cerebral ischemia. They can also prevent apoptosis in the cerebral ischemic tissue and promote angiogenesis as well as neurogenesis. These repair the damaged brain tissue via multiple mechanisms. The GM-CSF treatment can promote growth of collateral arterial branches in the pia mater and reduce the area of cerebral infarction due to arterial occlusion in the brain, thus protecting the nerves (Kim et al., 2012; Mukawa et al., 2012). Previous studies on cerebral ischemia have shown that multipoint skull drilling combined with mobilization of autologous BMSCs have a synergistic effect. These two interventions combined treat ischemic cerebrovascular diseases by preventing cell apoptosis and promoting vascular reconstruction and regeneration as well as the nerve regeneration repair mechanism (Kaku et al., 2012).

Statins are HMG-CoA reductase inhibitors. They regulate blood lipids and delay atherosclerosis. They also inhibit vascular endothelial inflammatory reactions and have other functions including anti-thrombus effect, stabilization of endothelial cells, and immune regulation, among others (Gurpinar et al., 2011; Deo et al., 2012). Recent studies have reported that simvastatin can also mobilize bone marrow endothelial stem cells, induce endogenous cell proliferation, enhance plasticity of nerve cells, promote vasculogenesis, and increase blood supply to the local tissues (Chen et al., 2009). It has also been reported that statins can improve nitric oxide synthase within the endothelial cells, activate antiplatelets, induce endogenous cell proliferation, enhance plasticity of nerve cells, promote vasculogenesis, maintain completeness of capillaries, increase blood supply to the local tissue, promote synaptic regeneration, and enhance repair of nerve injuries (Jabeen et al., 2011). Further, a previous study has found that statins can also mobilize bone marrow endothelial stem cells and significantly promote recovery of the nerve injury. Simvastatin can better penetrate the blood brain barrier and achieve an effective treatment concentration in the cerebrospinal fluid compared with other statins (Gholami et al., 2008). Combined application of BMSCs and statins can synergistically promote repair of nerve injuries and recovery of neurological functions. Research conducted previously has indicated that combined application of CSF and statins substantially promotes endogenous cell proliferation and improves nerve plasticity. This function may be associated with increased neurotrophic factors (Xavier et al., 2012). Studies on animals have indicated that multiple growth factors are generated after treatment with simvastatin. These include nerve growth factor (NGF), brain-derived neurotrophic factor (BDNF), and vascular endothelial cell growth factors significant to neural differentiation and regeneration. CSF pro- 
motes and induces BMSCs to secrete growth factors such as NGF and BDNF, whereas statins can increase secretion of vascular endothelial cell growth factors and BDNF (Liu et al., 2011). Therefore, the effect of their combined application is substantially improved. Cell growth factors affect neuron regeneration, synapse formation, and revascularization. The vascular endothelial cell growth factor is a type of revascularization factor that also promotes regeneration of nerve cells and axon growth. Combination of rhG-CSF/rhGM-CSF for differentiation and mobilization of autologous BMSCs and use of simvastatin can promote recovery of neurological functions after ischemic brain injury (Park et al., 2010).

Microsurgery combined with mobilization of autologous BMSCs and simvastatin has been proven to be a safe and effective treatment for hypertensive cerebral hemorrhage (McGirt et al., 2009). In the current study, indirect revascularization combined with mobilization of autologous stem cells and simvastatin was used for treatment of cerebral ischemia. According to the results, the improvement in patients' neurological functions was significantly superior in the treatment group that underwent indirect revascularization combined with autologous BMSCs and simvastatin compared to that in the treatment group with only indirect revascularization; the count of the CD133+CD34+ cells in the peripheral blood also increased significantly. None of the patients experienced any significant toxic effects or side effects during the entire study. This indicates that treating ischemic cerebrovascular diseases by indirect revascularization combined with autologous BMSC mobilization and simvastatin can promote recovery of neurological functions, reduce the disability rate of the patients with ischemic cerebrovascular disease, and improve the patients' daily living abilities and quality of life. It is, therefore, a safe and effective approach in the treatment of ischemic cerebrovascular diseases and worthy of clinical promotion.

\section{ACKNOWLEDGMENTS}

Research supported by the Natural Science Foundation of China (\#81360187, \#30960395, \#81000829), Natural Science Foundation of Inner Mongolia (\#MS1152, BS1102), and Shanghai Public Health Bureau Foundation (\#XYQ2011031).

\section{REFERENCES}

Almansob MA, Xu B, Zhou L, Hu XX, et al. (2012). Simvastatin reduces myocardial injury undergoing noncoronary artery cardiac surgery: a randomized controlled trial. Arterioscler. Thromb. Vasc. Biol. 32: 2304-2313.

Bao XY, Duan L, Li DS, Yang WZ, et al. (2012). Clinical features, surgical treatment and long-term outcome in adult patients with Moyamoya disease in China. Cerebrovasc. Dis. 34: 305-313.

Chen H, Ren JY, Xing Y, Zhang WL, et al. (2009). Short-term withdrawal of simvastatin induces endothelial dysfunction in patients with coronary artery disease: a dose-response effect dependent on endothelial nitric oxide synthase. Int. J. Cardiol. 131: 313-320.

Cho WS, Lee HY, Kang HS, Kim JE, et al. (2013). Symptomatic cerebral hyperperfusion on SPECT after indirect revascularization surgery for Moyamoya disease. Clin. Nucl. Med. 38: 44-46.

Choi IJ, Cho SJ, Chang JC, Park SQ, et al. (2012). Angiographic results of indirect and combined bypass surgery for adult moyamoya disease. J. Cerebrovasc. Endovasc. Neurosurg. 14: 216-222.

Deo SH, Fisher JP, Vianna LC, Kim A, et al. (2012). Statin therapy lowers muscle sympathetic nerve activity and oxidative stress in patients with heart failure. Am. J. Physiol. Heart Circ. Physiol. 303: 377-385.

Dorfman LJ, Fischbein NJ, Woodard JI, Choudhri O, et al. (2012). Moyamoya disease can masquerade as multiple sclerosis. Neurologist 18: 398-403.

Fujimoto S, Toyoda K, Inoue T, Jinnouchi J, et al. (2013). Changes in superficial temporal artery blood flow and cerebral hemodynamics after extracranial-intracranial bypass surgery in moyamoya disease and atherothrombotic carotid 
occlusion. J. Neurol. Sci. 325: 10-14.

Fujimura M, Inoue T, Shimizu H, Saito A, et al. (2012). Efficacy of prophylactic blood pressure lowering according to a standardized postoperative management protocol to prevent symptomatic cerebral hyperperfusion after direct revascularization surgery for moyamoya disease. Cerebrovasc. Dis. 33: 436-445.

Gholami MR, Abolhassani F, Pasbakhsh B, Akbari M, et al. (2008). The effects of simvastatin on ischemia-reperfusion injury of sciatic nerve in adult rats. Eur. J. Pharmacol. 590: 111-114.

Gu Y, Ni W, Jiang H, Ning G, et al. (2012). Efficacy of extracranial-intracranial revascularization for non-moyamoya steno-occlusive cerebrovascular disease in a series of 66 patients. J. Clin. Neurosci. 19: 1408-1415.

Gurpinar T, Ekerbicer N, Harzadin NU, Barut T, et al. (2011). Statin treatment reduces oxidative stress-associated apoptosis of sciatic nerve in diabetes mellitus. Biotech. Histochem. 86: 373-378.

Heijmen L, van Dijk EJ, Goraj B and van Laarhoven HW, et al. (2012). Moyamoya disease misdiagnosed as leptomeningeal metastases. J. Clin. Oncol. 30: e382-385.

Jabeen A, Khan UA and Lodhi GM (2011). Effects of simvastatin on lipid profile and nerve conduction velocity in obese Sprague Dawley rats. J. Ayub. Med. Coll. Abbottabad 23: 36-39.

Kainth DS, Chaudhry SA, Kainth HS, Suri FK, et al. (2013). Prevalence and characteristics of concurrent down syndrome in patients with moyamoya disease. Neurosurgery 72 : 210-215.

Kaku Y, Morioka M, Ohmori Y, Kawano T, et al. (2012). Outer-diameter narrowing of the internal carotid and middle cerebral arteries in moyamoya disease detected on 3D constructive interference in steady-state MR image: is arterial constrictive remodeling a major pathogenesis? Acta Neurochir. (Wien) 154: 2151-2157.

Kim HS, Lee SW, Sung SK and Seo EK (2012). Terson syndrome caused by intraventricular hemorrhage associated with moyamoya disease. J. Korean Neurosurg. Soc. 51: 367-369.

Kim JM, Jung KH, Sohn CH, Park J, et al. (2013). High-resolution MR technique can distinguish moyamoya disease from atherosclerotic occlusion. Neurology 80: 775-776.

Lee HM, Eun MY, Seo WK and Suh Si (2012). Thrombolysis for acute ischemic stroke in a patient with Moyamoya disease. Can. J. Neurol. Sci. 39: 687-689.

Liu ZQ, Liu B, Yu L, Wang XQ, et al. (2011). Simvastatin has beneficial effect on pulmonary artery hypertension by inhibiting NF-kappaB expression. Mol. Cell Biochem. 354: 77-82.

Liu X, Zhang D, Shuo W, Zhao Y, et al. (2013). Long term outcome after conservative and surgical treatment of haemorrhagic moyamoya disease. J. Neurol. Neurosurg. Psychiatry 84: 258-265.

McGirt MJ, Garces Ambrossi GL, Huang J, Tamargo RJ, et al. (2009). Simvastatin for the prevention of symptomatic cerebral vasospasm following aneurysmal subarachnoid hemorrhage: a single-institution prospective cohort study. J. Neurosurg. 110: 968-974.

Mishra A, Pendharkar H, Jayadaevan ER and Bodhey N (2012). Anomalous origins of bilateral vertebral arteries in a child with Down syndrome and Moyamoya disease. A case report. Interv. Neuroradiol. 18: 259-263.

Miyawaki S, Imai H, Takayanagi S, Mukasa A, et al. (2012). Identification of a genetic variant common to moyamoya disease and intracranial major artery stenosis/occlusion. Stroke 43: 3371-3374.

Motoshima S, Noguchi T, Kawashima M, Ooishi M, et al. (2012). Narrowed petrous carotid canal detection for the early diagnosis of moyamoya disease. Fukuoka Igaku Zasshi 103: 206-214.

Mukawa M, Nariai T, Matsushima Y, Tanaka Y, et al. (2012). Long-term follow-up of surgically treated juvenile patients with Moyamoya disease. J. Neurosurg. Pediatr. 10: 451-456.

Park JH, Park YS, Kim YJ, Lee IS, et al. (2010). Effects of statins on the epicardial fat thickness in patients with coronary artery stenosis underwent percutaneous coronary intervention: comparison of atorvastatin with simvastatin/ezetimibe. J. Cardiovasc. Ultrasound 18: 121-126.

Sasaki T, Fujimura M, Akamatsu Y, Kimura N, et al. (2012). A case of moyamoya disease presenting with progressive stroke during the late perinatal period successfully managed by bilateral revascularization surgeries. No Shinkei Geka 40: 1095-1099.

Skeik N, Rumery KK, Udayakumar PD, Crandall BM, et al. (2013). Concurrent takayasu arteritis with common variable immunodeficiency and moyamoya disease. Ann. Vasc. Surg. 27: e13-18.

Southerland AM, Meschia JF and Worrall BB (2013). Shared associations of nonatherosclerotic, large-vessel, cerebrovascular arteriopathies: considering intracranial aneurysms, cervical artery dissection, moyamoya disease and fibromuscular dysplasia. Curr. Opin. Neurol. 26: 13-28.

Takemoto Y, Morioka M, Nakagawa T, Hasegawa Y, et al. (2012). Prolonged and regionally progressive symptomatic cerebral hyperperfusion syndrome after superficial temporal artery-middle cerebral artery anastomosis in a patient with moyamoya disease. Surg. Neurol. Int. 3: 106.

Tseng LS and Luo SD (2013). Unilateral sudden hearing loss as the first presenting symptom of moyamoya disease. $J$. Laryngol. Otol. 127: 196-199. 
Uhiara CO, Alexander SP and Roberts RE (2012). Simvastatin evokes an unpredicted inhibition of beta-adrenoceptormediated vasodilatation in porcine coronary artery. Eur. J. Pharmacol. 690: 158-163.

Volkan-Salanci B, Lay Ergun E, Genc Sel C, Yalnizoglu D, et al. (2012). The role of brain perfusion SPECT in moyamoya disease. Rev. Esp. Med. Nucl. Imagen. Mol. 31:216-218.

Wang KC, Phi JH, Lee JY, Kim SK, et al. (2012). Indirect revascularization surgery for moyamoya disease in children and its special considerations. Korean J. Pediatr. 55: 408-413.

Xavier AM, Serafim KG, Higashi DT, Vanat N, et al. (2012). Simvastatin improves morphological and functional recovery of sciatic nerve injury in Wistar rats. Injury 43: 284-289.

Xu B, Song DL, Mao Y, Gu YX, et al. (2012). Superficial temporal artery-middle cerebral artery bypass combined with encephalo-duro-myo-synangiosis in treating moyamoya disease: surgical techniques, indications and midterm follow-up results. Chin. Med. J. (Engl) 125: 4398-4405.

Yonekawa Y (2012). Outer diameter measured by 3D CISS MRI and quasi-Moyamoya disease. Acta Neurochir. (Wien) 154: $2159-2161$. 